\title{
From Growth Surface to Device Interface: Preserving Metallic Fe under Monolayer Hexagonal Boron Nitride
}

Sabina Caneva, ${ }^{\dagger, \|}$ Marie-Blandine Martin, ${ }^{\dagger, \|}$ Lorenzo D’Arsié, ${ }^{\dagger}$ Adrianus I. Aria, ${ }^{\dagger, \perp}{ }^{\circ}$ Hikmet Sezen, ${ }^{\ddagger \oplus}$ Matteo Amati, ${ }^{\ddagger}$ Luca Gregoratti, ${ }^{\ddagger}$ Hisashi Sugime, ${ }^{\dagger}$ Santiago Esconjauregui, ${ }^{\dagger}$ John Robertson, ${ }^{\dagger}$ Stephan Hofmann, ${ }^{\dagger}{ }^{\circ}$ and Robert S. Weatherup ${ }^{*}, \S^{\circ}$ (1)

${ }^{\dagger}$ Department of Engineering, University of Cambridge, JJ Thomson Avenue, CB3 0FA Cambridge, U.K.

${ }^{\ddagger}$ Elettra-Sincrotrone Trieste S.C.p.A., AREA Science Park, S.S. 14 km 163.5, 34149 Trieste, Italy

${ }^{\S}$ Materials Sciences Division, Lawrence Berkeley National Laboratory, 1 Cyclotron Road, Berkeley, California 94720, United States

${ }^{\perp}$ Surface Engineering and Nanotechnology Institute, Cranfield University, College Road, MK43 OAL Cranfield, U.K.

Supporting Information

ABSTRACT: We investigate the interfacial chemistry between $\mathrm{Fe}$ catalyst foils and monolayer hexagonal boron nitride (h-BN) following chemical vapor deposition and during subsequent atmospheric exposure, using scanning electron microscopy, X-ray photoemission spectroscopy, and scanning photoelectron microscopy. We show that regions of the Fe surface covered by h-BN remain in a metallic state during exposure to moist air for $\sim 40 \mathrm{~h}$ at room temperature. This protection is attributed to the strong interfacial interaction between $\mathrm{h}-\mathrm{BN}$ and $\mathrm{Fe}$, which prevents the rapid intercalation
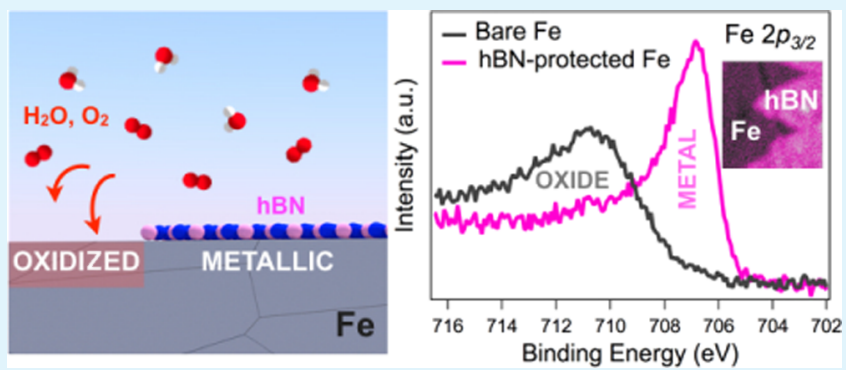
of oxidizing species. Local Fe oxidation is observed on bare $\mathrm{Fe}$ regions and close to defects in the h-BN film (e.g., domain boundaries, wrinkles, and edges), which over the longer-term provide pathways for slow bulk oxidation of Fe. We further confirm that the interface between h-BN and metallic Fe can be recovered by vacuum annealing at $\sim 600{ }^{\circ} \mathrm{C}$, although this is accompanied by the creation of defects within the h-BN film. We discuss the importance of these findings in the context of integrated manufacturing and transfer-free device integration of h-BN, particularly for technologically important applications where $\mathrm{h}-\mathrm{BN}$ has potential as a tunnel barrier such as magnetic tunnel junctions.

KEYWORDS: hexagonal boron nitride ( $h-B N)$, iron $(\mathrm{Fe})$, interfacial chemistry, X-ray photoelectron spectroscopy (XPS), chemical vapor deposition (CVD)

\section{INTRODUCTION}

Hexagonal boron nitride (h-BN), the isostructural and electrically insulating counterpart to graphene, is technologically promising not only as a support for high-mobility graphene and other two-dimensional (2D) materials ${ }^{1}$ but also, given its low permeability to gases and thermochemical stability, $^{2-4}$ as an ultimately thin barrier layer for integrated electronics, photonics, and spintronics. ${ }^{5,6}$ A critical requirement for numerous emerging device concepts is that, as well as the hBN layers being of high crystalline quality, their interface to other materials must be clean and well-controlled. Although significant progress has been made in $2 \mathrm{D}$ material crystal growth, ${ }^{7-9}$ particularly regarding scalable synthesis of h-BN films by chemical vapor deposition (CVD), ${ }^{10-14}$ clean interfacing and scalable device integration remain major challenges. The latter typically involves transfer of the atomically thin h-BN layers away from the CVD growth substrate; hence, the choice of CVD catalyst/substrate and the related interfacial chemistry must be optimized toward ease of release of the h-BN. ${ }^{15}$ Nevertheless, the potential for structural damage and contamination during transfer remains, ${ }^{16}$ and avoiding this step offers a cleaner and simpler process flow that is more amenable to integrated manufacturing.

Transfer-free h-BN integration requires direct, conformal hBN deposition onto a device-relevant substrate. This has been demonstrated for instance for vertical magnetic tunnel junctions (MTJs), where the bottom ferromagnetic device layer acts as a catalytic growth substrate for h-BN CVD. ${ }^{6}$ Although previous literature focused mainly on the quality of as-grown h-BN, crucial for this approach is also that the device substrate is not negatively affected by the CVD process and that the resulting interface remains stable during further processing. The latter links to the question of how effectively $2 \mathrm{D}$ materials can protect different substrates. For graphene, this has been investigated in detail and shown to relate to the strength of interaction between graphene and the substrate. ${ }^{17}$ Strongly interacting transition metals, such as $\mathrm{Ni}$ and $\mathrm{Fe}$, with a significant degree of hybridization between the graphene $\pi$

Received: June 16, 2017

Accepted: August 7, 2017

Published: August 7, 2017 
states and the $\mathrm{d}$ band states of the metal, ${ }^{18-20}$ prevent the rapid intercalation of oxidizing species at the graphene-metal interface and thus suppress oxidation and protect the metal surface. This protection can preserve clean, metallic interfaces, which is a crucial requirement for effective spin injectors, and the importance of this has been demonstrated for graphenebased MTJs. ${ }^{21-23}$ Recent literature highlights that h-BN as a tunnel barrier may outperform widely used oxide barriers ${ }^{24,25}$ and when paired with a metallic ferromagnet can allow effective spin-filtering. ${ }^{6,26-29}$ A tunnel magnetoresistance of up to $6 \%$ has already been demonstrated for a MTJ based on a single h$\mathrm{BN}$ layer grown directly on the Fe electrode. ${ }^{6}$ Although highquality h-BN CVD on Fe has been reported and it has been shown how boride and nitride formation in the Fe bulk can be avoided, ${ }^{10,11}$ the nature and stability of as-grown h-BN/Fe interfaces, which are of utmost importance to device integration, have not been studied in detail.

Here, we use scanning electron microscopy (SEM), X-ray photoemission spectroscopy (XPS), and scanning photoelectron microscopy (SPEM) to systematically study the interfacial chemistry between $\mathrm{Fe}$ and h-BN after CVD and during subsequent atmospheric exposure. This model system has been chosen based on its particular relevance to MTJ device applications. Our SPEM data reveals that the Fe under h-BN is protected from oxidation under ambient conditions for several days. The single-layer h-BN provides a low-permeability barrier that restricts the access of oxidizing species to the metal surface, and the strong interaction between $\mathrm{h}-\mathrm{BN}$ and $\mathrm{Fe}$ prevents the rapid intercalation of oxidizing species at the $h-B N / F e$ interface. However, $\mathrm{Fe}$ does not form a passivating oxide when exposed to moist air; therefore, during continuing exposure, the slow oxidation of the metal can proceed through the oxide layers formed in the vicinity of h-BN edges and defects. We further demonstrate that a h-BN/metallic-Fe interface can be recovered by vacuum annealing of a sample exposed to air for 7 days, with the reduction of Fe confirmed by XPS. We find that the general rationale for effective passivation by h-BN is consistent with a model developed for graphene ${ }^{17}$ and discuss the importance of our findings in the context of transfer-free h-BN device integration and MTJ applications where the protection demonstrated ensures a window for device processing in air and other oxidizing environments.

\section{RESULTS}

We investigate the time-dependent oxidation behavior of polycrystalline $\mathrm{Fe}$ foils (100 $\mu \mathrm{m}$ thick) that have single-layer h-BN grown directly on their surface by CVD and are subsequently exposed to atmospheric air at room temperature. All growths are performed in a customized CVD reactor (base pressure $10^{-6} \mathrm{mbar}$ ) with the $\mathrm{Fe}$ foils preannealed in $\mathrm{NH}_{3}(4$ mbar) at $\sim 900{ }^{\circ} \mathrm{C}$ prior to $\mathrm{B}_{3} \mathrm{~N}_{3} \mathrm{H}_{6}$ exposure at the same temperature. The preannealing enriches the Fe bulk with $\mathrm{N}$, promoting the exclusive growth of single-layer h-BN on the catalyst surface and avoiding any Fe-boride formation. ${ }^{11}$

Figure 1 compares SEM images of discontinuous domains $(a-c)$ and complete films $(\mathrm{d}-\mathrm{f})$ of single-layer h-BN on the Fe catalyst that have been exposed to air for different times following growth. The dark triangles in Figure 1a correspond to single-layer h-BN after $30 \mathrm{~min}$ of air exposure. After 1 day, thin bright veins can be observed crossing the domains (Figure $1 \mathrm{~b}$ ). We have previously shown by dark field transmission electron microscopy that the individual, triangular $\mathrm{h}-\mathrm{BN}$ islands are single crystals; ${ }^{11}$ therefore, the bright veins are expected to

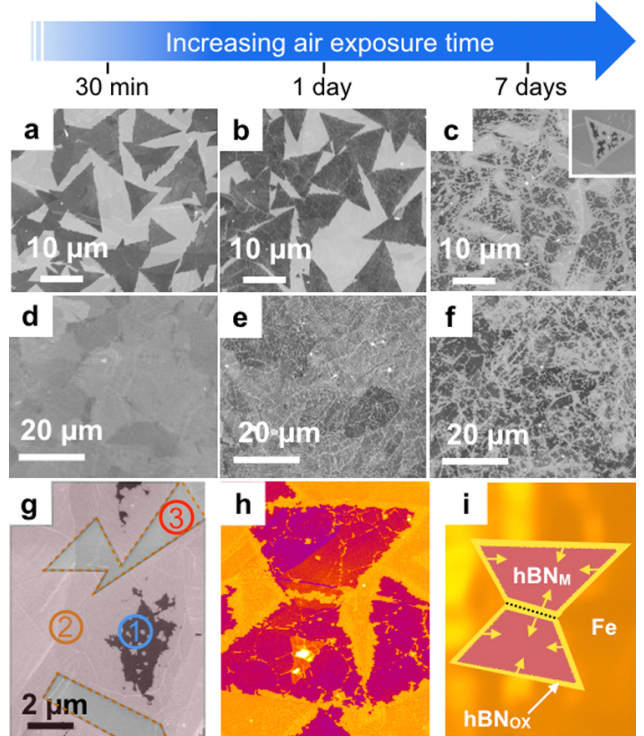

Figure 1. $(\mathrm{a}-\mathrm{c}) \mathrm{h}-\mathrm{BN}$ domains on the Fe substrate after $30 \mathrm{~min}, 1$ day, and 7 days of air exposure, respectively. (d-f) h-BN film on the $\mathrm{Fe}$ substrate after $30 \mathrm{~min}, 1$ day, and 7 days of air exposure, respectively. h-BN was grown at $\sim 900{ }^{\circ} \mathrm{C}$, by $\mathrm{B}_{3} \mathrm{~N}_{3} \mathrm{H}_{6}\left(6 \times 10^{-4} \mathrm{mbar}\right)$ exposure for $45 \mathrm{~s}$ (domains) or $12 \mathrm{~min}$ (films). (g) Detail of merging h-BN domains (left, right, and top) after 7 days of air exposure, illustrating a contrast difference between the middle of the domains (black, region 1 ), domain edges (light gray, region 2), and the uncovered Fe catalyst (dark gray, region 3). (h) False color SEM image showing the detail of bow-tie-shaped h-BN domains after 1 day of air exposure and (i) corresponding schematic indicating that the change of contrast initiates at the domain edges and domain boundaries (dotted line) and proceeds inward. $\mathrm{h}-\mathrm{B}_{\mathrm{NM}}$ and $\mathrm{h}-\mathrm{BN}_{\mathrm{Ox}}$ indicate $\mathrm{h}-\mathrm{BN}$ covering metallic and oxidized Fe regions, respectively.

relate to wrinkling of $\mathrm{h}-\mathrm{BN}$ caused by the thermal expansion mismatch with the underlying $\mathrm{Fe}$ rather than to domain boundaries in h-BN. ${ }^{30}$ After 7 days in air, the brighter regions are found to have grown at the expense of the darker regions within the domains. The inset shows a high-magnification image of a small isolated h-BN domain, which reveals a band of brighter contrast around the perimeter, indicating that the contrast change proceeds inward from the edges. Figure $1 \mathrm{~d}-\mathrm{f}$ shows the morphology of a complete single-layer h-BN film (i.e., without bare $\mathrm{Fe}$ regions) after $30 \mathrm{~min}, 1$ day, and 7 days of air exposure. The SEM contrast is again seen to evolve over time, from dark to bright. Following $30 \mathrm{~min}$ of air exposure, the h-BN-related contrast is uniform and dark within each Fe grain. We note that the relatively minor grain-to-grain contrast variations of the $\mathrm{Fe}$ catalyst are related to the electron channeling contrast routinely observed for polycrystalline metal surfaces and do not reflect a variation in the thickness of $h$ $\mathrm{BN}^{31}$ After 1 day, we again observe bright lines spreading across the surface, and after 7 days, the bright regions surround the few dark areas remaining. Figure $S 1$ shows in more detail a representative area of the h-BN film after 1 day in air, where a network of brighter channels is apparent, from which the change in secondary electron contrast proceeds. Given the shapes and distribution of these channels, they are expected to correspond with the network of wrinkles and domain boundaries within the h-BN film.

Figure $1 \mathrm{~g}$ shows in detail the edges and tips of several partially merged h-BN domains where different levels of contrast can be distinguished, labeled as regions $1-3$. It is 

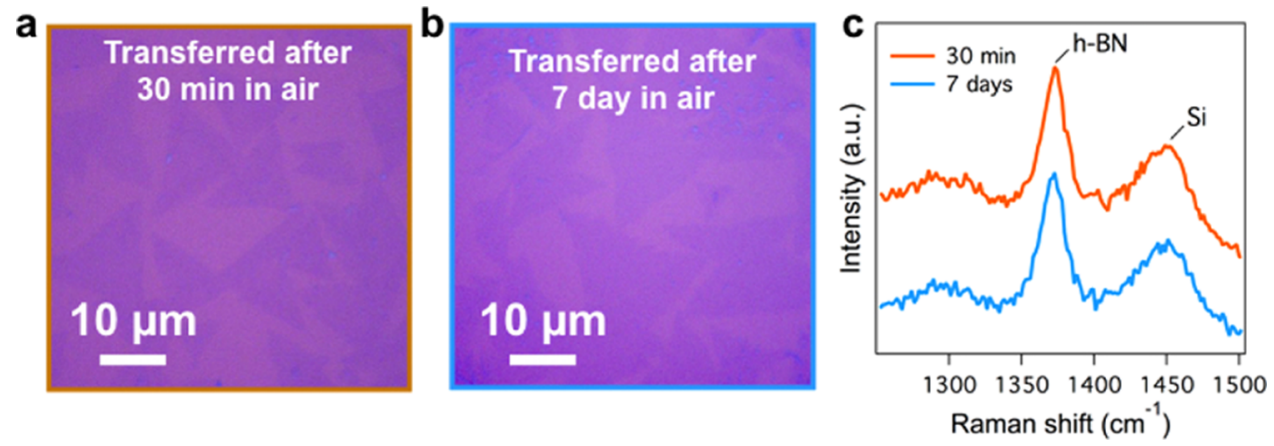

Figure 2. Optical images after transfer onto $\mathrm{SiO}_{2}(300 \mathrm{~nm}) / \mathrm{Si}$ of (a) fresh h-BN domains (transferred directly after growth) and (b) h-BN domains exposed to air for 7 days and then transferred, showing that the triangular shape and size is preserved for both air exposure times. (c) Raman spectra of the samples in (a) and (b) displaying the characteristic peak of $\mathrm{h}-\mathrm{BN}$ at $\sim 1370 \mathrm{~cm}^{-1}$ and the Si substrate-related peak. ${ }^{60}$

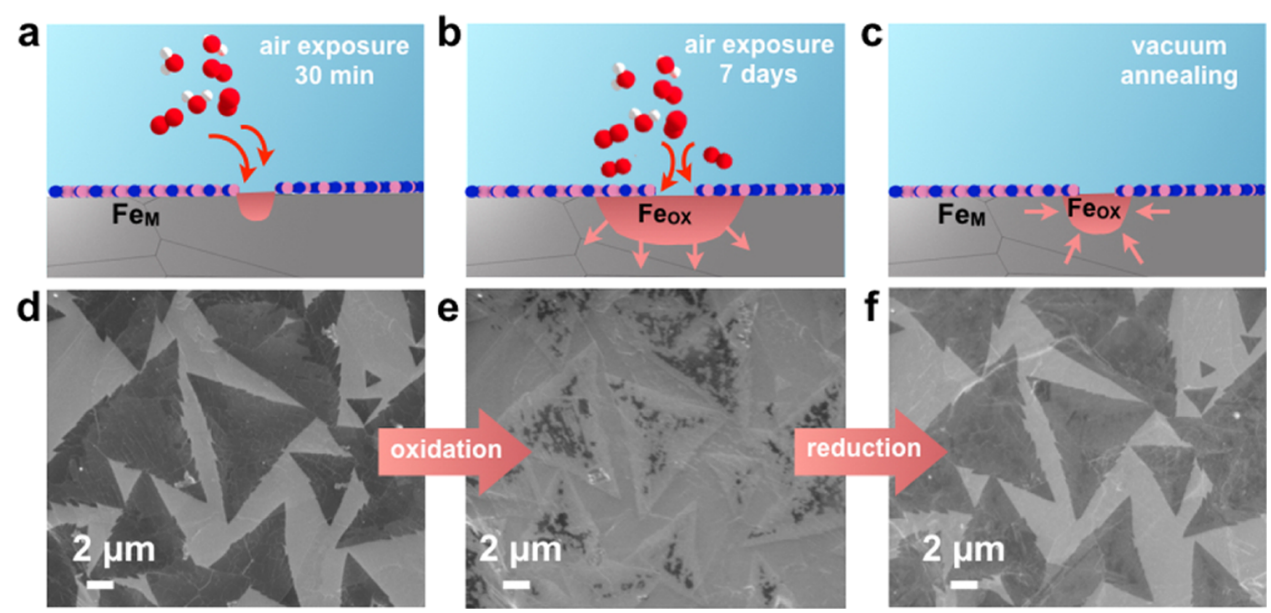

Figure 3. Schematics illustrating single-layer h-BN on Fe foil (a) after a short air exposure, showing initial traces of Fe oxidation, close to h-BN defect sites or domain edges (pink area), (b) after 7 days in air, showing gradual oxidation of Fe through the catalyst bulk, and (c) during annealing in vacuum at $\sim 600^{\circ} \mathrm{C}$ for $1 \mathrm{~h}$ showing reduction of oxidized Fe. $(\mathrm{d}-\mathrm{f})$ Corresponding SEM images of the samples described in $(\mathrm{a}-\mathrm{c})$ showing a fresh sample (30 min air exposure), the same sample after 7 days air exposure, and again following vacuum annealing.

interesting to note that the brightest regions (region 2, shaded red), although having lost their initial dark contrast (i.e., region 1 ), still bear the shape of the sharp h-BN domain edges, which can be distinguished from the areas of bare $\mathrm{Fe}$ (region 3 ). Figure $1 \mathrm{~h}$ shows a false-colored SEM image of a "bow tie" h-BN domain structure, where two triangular domains have partially coalesced, creating a domain boundary at the merging front. The domain edges and the domain-boundary region display a different contrast, suggesting that the contrast changes proceed inward from locations at which the atmosphere can more easily access the Fe foil, such as through edges and the defects present at boundaries (Figure 1i). ${ }^{31,32}$

We confirm that the change in SEM contrast is not indicative of a loss of h-BN material by transferring fresh h-BN domains (30 min air exposure) and aged domains (7 day air exposure) onto $\mathrm{SiO}_{2}(300 \mathrm{~nm}) / \mathrm{Si}$ wafers. The optical images in Figure $2 \mathrm{a}, \mathrm{b}$ demonstrate that the triangular shapes and sizes of the $\mathrm{h}$ $\mathrm{BN}$ domains are preserved and are very similar to those of the domains grown on the catalyst (Figure 1a). The corresponding Raman spectra (Figure 2c) show very similar signatures for both samples, confirming that h-BN is present on both and retains similar structural quality insofar as Raman spectroscopy is sensitive to this. Additionally, the XP B 1s and N 1s core-level spectra for a nontransferred sample (i.e., h-BN/Fe) exposed to air for $\sim 40 \mathrm{~h}$ (Figure S2) exhibit a dominant B/N peak pair at $190.2 / 397.8 \mathrm{eV}$, which can be attributed to high-quality monolayer h-BN. ${ }^{11}$ This combined with the absence of any peak at $\sim 193 \mathrm{eV}$ in the B 1s core level, the typical position of boron oxide species, ${ }^{33}$ indicates that the intrinsic chemical structure of h-BN is not significantly altered by atmospheric air exposure.

Further evidence that $\mathrm{h}-\mathrm{BN}$ is stable in air at room temperature over time is obtained without a transfer step, by vacuum annealing $\left[\sim 600^{\circ} \mathrm{C}\right.$ for $\left.1 \mathrm{~h}\left(<10^{-5} \mathrm{mbar}\right)\right]$ of a sample after 7 days of air exposure, as illustrated in Figure $3 a-f$. The behavior observed is schematically summarized in $(a)-(c)$, with the corresponding SEM images shown in (d)-(f). At the onset of air exposure, the h-BN domains on the metal surface show relatively homogeneous dark contrast (Figure 3a,d). With continued air exposure, this contrast is gradually lost (Figure $3 \mathrm{~b}, \mathrm{e})$. On vacuum annealing at $\sim 600{ }^{\circ} \mathrm{C}$, the domains recover dark contrast similar to that observed for the fresh h-BN samples immediately after CVD (Figure 3c,f), further demonstrating that despite the changes in contrast h-BN remains intact. In situ XPS measurements of similar samples during vacuum annealing at $\sim 600{ }^{\circ} \mathrm{C}$ show no emergence of a boron oxide-related peak at $\sim 193 \mathrm{eV}$ nor any detectable oxygen species in the $\mathrm{O} 1 \mathrm{~s}$ region, confirming the effective reduction of Fe without oxidation of h-BN (see Figure S3). However, XPS measurements after a further air exposure reveal some oxidation of the h-BN films, as manifested by the appearance of a boron oxide peak at $\sim 193 \mathrm{eV}$. This therefore indicates that the 
annealing process has led to some degradation of $\mathrm{h}-\mathrm{BN}$, with defects formed, which are then oxidized on air exposure.

Figure $4 \mathrm{a}, \mathrm{b}$ shows XP spectra of the $\mathrm{Fe} 2 \mathrm{p}_{3 / 2}$ core level for a complete h-BN film and discontinuous domains, respectively as

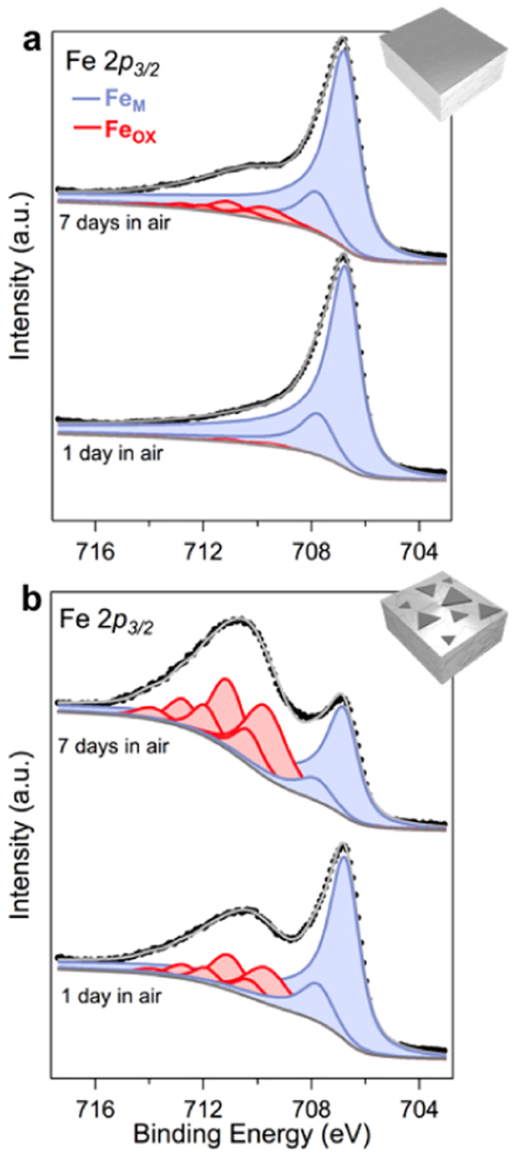

Figure 4. XP spectra of the $\mathrm{Fe} 2 \mathrm{p}_{3 / 2}$ core level for (a) a continuous single-layer h-BN film and (b) discontinuous single-layer h-BN domains as a function of air exposure time ( 1 day and 7 days). We note that the level of oxidation in (b) does not imply that the domains do not protect $\mathrm{Fe}$, as the spectra are acquired from a $\sim 150 \mu \mathrm{m}^{2}$ area of the sample, which includes both $\mathrm{h}-\mathrm{BN}$-covered and uncovered regions. The spectra are collected at a photon energy, $E_{\text {photon }}$ of $1486.6 \mathrm{eV}$.

a function of air exposure time. After 1 day in air, the spectrum for the complete film displays a dominant metallic peak at 706.7 $\mathrm{eV}\left(\mathrm{Fe}_{\mathrm{M}}\right)$ and negligible oxide-related components, indicating that the Fe is well reduced. After 7 days, Fe is still mainly in the reduced state; however, a small peak at the oxide position emerges above the noise level, at $\sim 711 \mathrm{eV}\left(\mathrm{Fe}_{\mathrm{OX}}\right)$, which is indicative of slight oxidation. This behavior is in strong contrast to that of bare $\mathrm{Fe}$, where significant oxidation of the surface is observed following air exposures of just $1 \mathrm{~h}$ but is in excellent agreement with our previous studies on air-exposed graphenecoated Fe substrates, where gradual oxidation of the metal is observed. ${ }^{17}$ For discontinuous h-BN domains (Figure 4b), the spectral components related to both metallic and oxidized $\mathrm{Fe}$ are observed following 1 day of air exposure. The metallic peak dominates over the oxide-related peak, indicating that $\mathrm{Fe}$ remains predominantly reduced, consistent with the fact that only the exposed $\mathrm{Fe}$ regions oxidize, whereas the h-BN-covered regions remain reduced. However, after 7 days, the extent of $\mathrm{Fe}$ oxidation is significantly increased, as revealed by the greater intensity of the oxide components relative to the metal peak.
Figure 5a shows a SPEM map of h-BN domains grown on $\mathrm{Fe}$ foil (same growth conditions as in Figure 1a) acquired for the B 1s core-level energy, following exposure to atmospheric air for $\sim 40 \mathrm{~h}$ at room temperature. The schematic in the inset indicates that the light gray triangles correspond to h-BN domains and the surrounding dark areas correspond to the bare Fe surface, as subsequently confirmed by the maps in Figure $5 c$,d. XP spectra of the Fe $2 p_{3 / 2}$ core level were acquired on the bare $\mathrm{Fe}$ (region $\mathrm{A}$ ) and on a h-BN domain (region $\mathrm{B}$ ), as shown in Figure $5 \mathrm{~b}$. The change in spectral components present in these two regions indicates a distinct difference in the oxidation state of $\mathrm{Fe}$ between the h-BN-covered regions and bare Fe.

The peaks from region $\mathrm{A}$ are attributable to the Fe oxide/ oxyhydroxide species $\left(\mathrm{Fe}_{\mathrm{OX}}\right),{ }^{34}$ whereas the peaks from region $\mathrm{B}$ correspond to metallic $\mathrm{Fe}\left(\mathrm{Fe}_{\mathrm{M}}\right)$. Figure $5 \mathrm{c}-\mathrm{e}$ shows the elemental maps of the edge and tips of several h-BN domains and the neighboring bare Fe surface using the $\mathrm{B} 1 \mathrm{~s}$ and $\mathrm{N}$ 1s peak intensities and the intensity ratio between the metal and oxide components in the $\mathrm{Fe} 2 \mathrm{p}_{3 / 2}$ region, respectively. In the $\mathrm{B}$ $1 \mathrm{~s}$ and $\mathrm{N} 1 \mathrm{~s}$ maps, the right-hand side of the image appears brighter, indicating a higher signal originating from this area, which confirms h-BN coverage of this region. We note that a Fe grain boundary can be seen crossing slightly diagonally from the top to the bottom of Figure 5c,d across several h-BN domains, which is apparent due to topographic changes in the $\mathrm{XP}$ signal. Figure 5e, which depicts a map of the ratio between the metal and oxide components of the $\mathrm{Fe} 2 \mathrm{p}_{3 / 2}$ core level, is a background-corrected chemical map (see Methods) where the intensity changes generated by the topography of the sample are intrinsically removed, thus revealing only chemical data. The brightest contrast, that is the predominantly metallic Fe, corresponds to the h-BN-covered region on the right-hand side, confirming that oxidation protection arises from the h-BN coverage. The fact that the contrast level is also maintained across the Fe grain boundary indicates that the extent of protection afforded by the h-BN layer is maintained in spite of the topography of the underlying substrate. Where h-BN is absent on the left-hand side of Figure 1e, the signal is less bright, confirming that the uncovered $\mathrm{Fe}$ is predominantly oxidized. The $\mathrm{B} 1 \mathrm{~s}$ and $\mathrm{N} 1 \mathrm{~s}$ chemical maps with the topographic component removed are shown in Figure S4.

\section{DISCUSSION}

The changes in SEM contrast observed in Figure 1 may at first sight appear to correspond to degradation of h-BN in air; however, our XPS and SPEM data confirm that they instead relate to the gradual oxidation of the Fe substrate beneath hBN. Similar contrast changes are observed for graphene on weakly interacting metals due to the intercalation of oxidizing species, which alter the charge-transfer doping from the underlying substrate and thus the work function of the graphene, without otherwise significantly disturbing its band structure. $^{17,35}$ In the case of $\mathrm{h}-\mathrm{BN}$ on $\mathrm{Fe}$, intercalation is suppressed due to the strong interaction between h-BN and the substrate, but as our XPS measurements here show, oxidation can still proceed through the Fe bulk. The changes in SEM contrast observed for $\mathrm{h}-\mathrm{BN}$-covered regions are therefore attributable to the different secondary electron yields of oxidized and metallic $\mathrm{Fe}$, as well as possible variations in the work function associated with changes in hybridization between h-BN and the substrate (i.e., alteration of the h-BN band structure $^{36-38}$ ) as the substrate oxidizes. The changes in the 
a

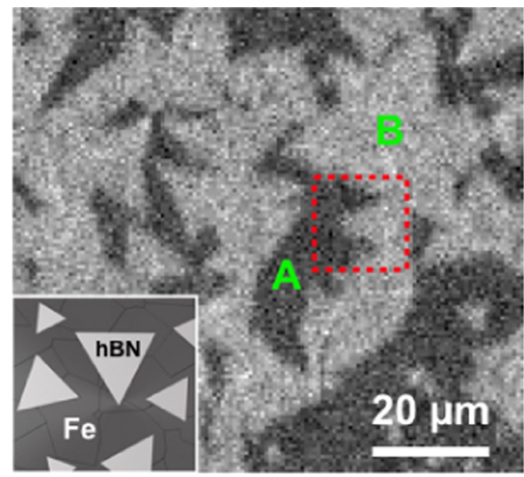

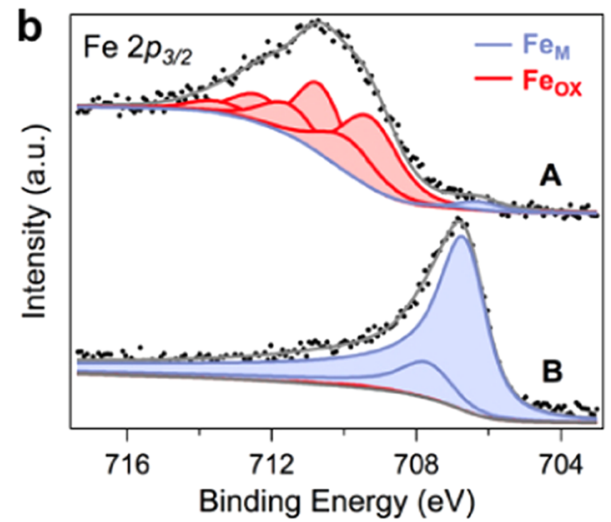
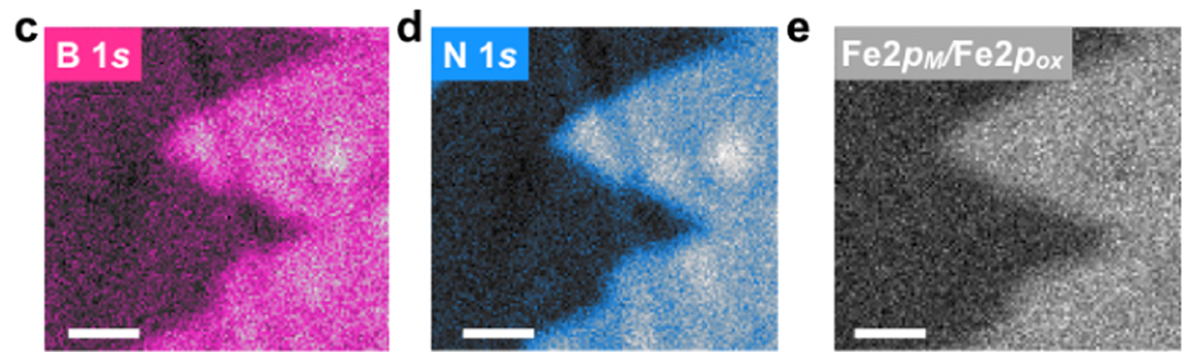

Figure 5. (a) Scanning photoemission spectroscopy (SPEM) map using the B 1s core-level energy, showing h-BN domains grown on Fe foil [ 900 ${ }^{\circ} \mathrm{C}, \mathrm{B}_{3} \mathrm{~N}_{3} \mathrm{H}_{6}\left(6 \times 10^{-4} \mathrm{mbar}\right)$ for $45 \mathrm{~s}$, air exposed for $\sim 40 \mathrm{~h}$ ]. Inset: schematic illustrating that the light gray triangles correspond to single-layer h$\mathrm{BN}$ and the surrounding dark regions correspond to the bare Fe surface. (b) Microfocussed XP spectra of the Fe $2 \mathrm{p}_{3 / 2}$ core level acquired on the bare Fe (region A) and on a h-BN domain (region B). The SPEM map of the tips and edges of several h-BN domains from the region within the red dotted box acquired with (c) B 1s and (d) N 1s core-level energies, and (e) chemical information without topography of the Fe $2 \mathrm{p}_{3 / 2}$ metal/oxide peak area ratio. The scalebars in (c) $-(\mathrm{e})$ are $2 \mu \mathrm{m}$. The Fe $2 \mathrm{p}_{3 / 2} \mathrm{~B} 1 \mathrm{~s}$ and $\mathrm{N} 1 \mathrm{~s}$ spectra are collected at a photon energy, $E_{\text {photon }}$ of $1102 \mathrm{eV}$.

SEM contrast from dark to light with continuing air exposure are thus indicative of oxidation of the underlying metal. We can therefore assign the different contrast levels in Figure $1 \mathrm{~g}$ to h$\mathrm{BN}$-covered metallic $\mathrm{Fe}\left(\mathrm{h}-\mathrm{BN} / \mathrm{Fe}_{\mathrm{M}}\right)$ in region 1 , h-BN-covered oxidized $\mathrm{Fe}\left(\mathrm{h}-\mathrm{BN} / \mathrm{Fe}_{\mathrm{OX}}\right)$ in region 2 , and bare $\mathrm{Fe}$ in region 3. The contrast changes apparent in Figure 1 thus correspond to $\mathrm{Fe}$ oxidation proceeding via domain edges, domain-boundary defects, and wrinkles of the h-BN layer, which serve as pathways for oxidizing species to reach the Fe surface during atmospheric air exposure. Given the detrimental effect of wrinkles on the passivation behavior of $2 \mathrm{D}$ films, we envisage that future work could be aimed at minimizing wrinkle formation during CVD growth by, for instance, varying the thickness of the catalyst, as has been reported for graphene CVD. $^{39}$

The XPS spectra shown in Figure 4, acquired using a X-ray spot size of $\sim 150 \mu \mathrm{m}$, further confirm that oxidation proceeds through these pathways. In the case of the continuous h-BN film, the region probed is fully covered with h-BN and Figure 4a clearly confirms that $\mathrm{Fe}$ is protected from any substantial oxidation over the course of 1 day and that even after 7 days of air exposure the level of oxidation remains relatively low. For the discontinuous domains, the level of oxidation after 1 day is significantly increased, showing that the degree of protection depends on the extent of h-BN coverage. Furthermore, the increase in oxidation after 7 days is much more pronounced than for the continuous h-BN film, indicating that the increased prevalence of "open edges" allows oxidation to proceed more readily. However, as the XPS spot includes both covered and uncovered regions, we note that Figure $4 \mathrm{~b}$ does not necessarily imply that the discontinuous h-BN domains do not locally protect Fe. This point is clarified by Figure 5 where spatially resolved XP spectra of the Fe $2 \mathrm{p}_{3 / 2}$ core level reveal that in the short term $(\sim 40 \mathrm{~h})$ significant oxidation $\left(\mathrm{Fe}_{\mathrm{OX}}\right)$ is observed only in the regions of the surface that are not covered by h-BN, with the h-BN-covered regions remaining reduced $\left(\mathrm{Fe}_{\mathrm{M}}\right)$.

The observed oxidation behavior can be understood in the context of the model recently introduced by Weatherup et al. ${ }^{17}$ to explain the behavior of different graphene/metal couples as a function of their interfacial interaction strength and the nature of the oxide formed by the substrate. Here, we extend this model to h-BN on Fe, as summarized in Figure 3, highlighting its general applicability to other 2D materials. During initial exposure to atmospheric air at room temperature, h-BN grown on $\mathrm{Fe}$ provides a low-permeability barrier that restricts the access of oxidizing species to the underlying metal. Furthermore, the strong interaction between h-BN and $\mathrm{Fe}$ prevents intercalation of oxidizing species at the $\mathrm{h}-\mathrm{BN}-$ metal interface. Rapid oxidation thus occurs only at uncovered regions or close to intrinsic defects in the h-BN film (e.g., vacancies, grain boundaries) that provide pathways for oxidizing species to reach the substrate (shaded red, Figure 3a), whereas the areas with pristine $h-B N$ coverage remain reduced $\left(\mathrm{Fe}_{\mathrm{M}}\right)$. As discussed further below, $\mathrm{Fe}$ does not form a passivating oxide when exposed to moist air. ${ }^{40}$ Therefore, during continuing exposure (days), oxidation can proceed through the oxide layers formed in the vicinity of h-BN edges and defects (expanding $\mathrm{Fe}_{\mathrm{OX}}$ region underneath the strongly coupled h-BN in Figure $3 \mathrm{~b}$ ), with $\mathrm{Fe}$ eventually becoming oxidized throughout. This process can be subsequently reversed by vacuum annealing, which leads to reduction of the Fe layer (Figure 3c). Our results herein for h-BN and previous work on graphene $^{17}$ emphasize the importance of the interfacial interaction strength in how effectively a $2 \mathrm{D}$ material protects 
a substrate. Therefore, we expect the development of methods to engineer this interfacial interaction strength, through techniques such as alloying or interfacial intercalation, to be a promising avenue for protecting material surfaces while retaining their functional (e.g., catalytic, ${ }^{41,42}$ plasmonic, ${ }^{43}$ and spin-filtering ${ }^{6,21-23}$ ) properties.

In this work, we employ polycrystalline $\mathrm{Fe}$ foils comprising multiple crystal orientations and note that the h-BN domains readily grow across $\mathrm{Fe}$ grain boundaries. Furthermore, Fe can undergo a range of phase transformations and reactions with boron and nitrogen during the CVD process including upon cooling. ${ }^{11}$ The nature of the edge bonding and any epitaxial relationship will therefore vary across different Fe grains, within $\mathrm{h}-\mathrm{BN}$ domains, and at different stages of the growth process, precluding here a simple explanation of the local oxidation behavior based on the interlayer distance, epitaxial relationship, or edge bonding state. The formation of wrinkles in the h-BN layer, which provide pathways between $\mathrm{h}-\mathrm{BN}$ and $\mathrm{Fe}$ for more rapid surface oxidation, is also likely to be impacted by the complex phase behavior that the $\mathrm{Fe}$ surfaces undergo. Interestingly, we note that the general oxidation behavior of regions away from wrinkles appears similar across multiple hBN domains on several samples. This indicates that the firstorder picture of the oxidation behavior we develop, based on a strong $\mathrm{Fe}$ and h-BN hybridization preventing rapid intercalation of oxidizing species, is relevant across such complex polycrystalline surfaces.

Previous XPS studies on the behavior of polycrystalline $\mathrm{Fe}$ surfaces exposed to either oxygen or water vapor have elucidated the various stages of the oxidation mechanisms. ${ }^{44,45}$ In both cases, current interpretation suggests that a thin surface oxide initially forms by a place-exchange mechanism and then growth of the oxide proceeds by electric-field-driven diffusion of ions through vacancies, interstitial sites, and grain boundaries. As the oxide thickness increases, the electric field strength reduces, limiting the oxide thickness to several nanometers. A slower rate of oxide thickening is observed for $\mathrm{H}_{2} \mathrm{O}$ compared to that for $\mathrm{O}_{2}$, which is attributable to the presence of hydrogen, which restricts ion diffusion and occupies surface sites that would otherwise be available for further $\mathrm{H}_{2} \mathrm{O}$ adsorption. ${ }^{45}$ When bare $\mathrm{Fe}$ is instead exposed to moist air containing both $\mathrm{O}_{2}$ and $\mathrm{H}_{2} \mathrm{O}$, a hydrated oxide forms as a loose deposit that provides very limited protection against further oxidation. $^{40}$ Therefore, whereas a h-BN overlayer prevents oxidation of the Fe surface beneath for several days by acting as an impermeable barrier, $\mathrm{Fe}$ does not form an effective passivating oxide that can "plug" regions near h-BN defects and edges and therefore oxidation proceeds through the $\mathrm{Fe}$ bulk over the longer term.

In contrast to graphene-covered metals, h-BN is not expected to promote galvanic corrosion despite the presence of moist air, as its insulating nature prevents electron conduction to the oxygen atoms. However, the presence of h-BN edges and the confined space between the metal and h-BN may potentially influence the passivation behavior. For metal substrates including $\mathrm{Pt}, \mathrm{Rh}$, and $\mathrm{Ru}$, confinement of molecules beneath a $2 \mathrm{D}$ layer may enhance metal-catalyzed reactions by modifying the adsorbate-metal interaction and potentially causing reconstruction of the metal surface. ${ }^{46-48}$ In our case, however, the strong interaction between $\mathrm{Fe}$ and $\mathrm{h}-\mathrm{BN}$ suppresses intercalation of molecules; therefore, we suggest that any change in the reactivity of Fe caused by h-BN is likely to occur only locally at the h-BN wrinkles or grain boundaries, which constitute a small proportion of the total surface.

A key consideration in selecting a passivating layer is its stability in its intended operating environment. For certain 2D materials, including $\mathrm{MoS}_{2}, \mathrm{WS}_{2}$, and black phosphorous, significant structural degradation has been reported under atmospheric conditions at room temperature. ${ }^{49-51}$ However, h$\mathrm{BN}$ is expected to exhibit excellent stability under atmospheric conditions, and indeed the optical images and Raman spectra we obtain (Figure 2) confirm that the h-BN on $\mathrm{Fe}$ does not undergo significant deterioration of its structural quality during room temperature air exposure. We further exclude significant defect formation within the domains after room temperature air exposure based on the absence of any component in the XP spectra at $\sim 193 \mathrm{eV}$ (Figure S2), which is typically attributed to boron oxide species ${ }^{33,52}$ whose formation has been linked to the loss of $\mathrm{N}$ atoms from the h-BN lattice. ${ }^{53}$ This excellent stability in air is also evidenced by the recovery of the initial, dark SEM contrast in h-BN-covered regions after vacuum annealing (Figure 3f). The reduction of the underlying Fe that occurs during this process (as confirmed by XPS in Figure S3) is achieved without accompanying structural damage being detected with SEM. In contrast, SEM following vacuum annealing of air-exposed graphene on $\mathrm{Cu}$ and h-BN on $\mathrm{Cu}$ reveals that significant etching of the $2 \mathrm{D}$ materials occurs. In situ XPS confirms that this happens at temperature by carbothermal reduction of the oxidized $\mathrm{Cu}$ substrate for graphene, ${ }^{35}$ and by formation of volatile boron oxides for $\mathrm{h}$ $\mathrm{BN}$, with the extent of damage linked to the amount of substrate oxidation and oxygen species present at the interface as well as the catalytic nature of the substrate (including crystal orientation). ${ }^{52} \mathrm{On} \mathrm{Cu}$, which exhibits a weak interaction with graphene and h-BN, rapid decoupling on air exposure allows oxidizing species to readily access the $\mathrm{Cu}$ and thus a relatively large oxygen reservoir for etching can develop. Our in situ measurements on $\mathrm{Fe}$ herein show no detectable boron oxide species (either in the $\mathrm{B} 1 \mathrm{~s}$ or $\mathrm{O}$ 1s spectra) during annealing (Figure S3). Nevertheless, we do find that annealing induces some minor degradation of $\mathrm{h}-\mathrm{BN}$, as evidenced by the appearance of a boron oxide peak in the B 1s spectrum following subsequent air exposure (Figure S3c), which we attribute to the decoration of defects induced in h-BN during annealing. This indicates that catalytic etching of h-BN on $\mathrm{Fe}$ can still occur but that this is relatively minor as only a small oxygen reservoir is formed over the time scales considered here thanks to the strong interfacial interaction that suppresses $\mathrm{Fe}$ oxidation. However, this does call into question the viability of using thin h-BN layers as oxidation-resistant coatings under high-temperature conditions, ${ }^{3}$ as the potential for catalytic degradation of h-BN by the substrate it is protecting must be carefully considered. We emphasize that the postannealed $\mathrm{Fe}$ surface remains reduced during subsequent exposure to air, as shown in Figure 3f, and hence demonstrate that protection can be re-established through this treatment.

Our results demonstrate that single-layer h-BN can protect Fe from oxidation for several days without undergoing any significant degradation. Although the protection afforded is not superior to that of graphene-covered $\mathrm{Ni}$ substrates, which remain reduced for $>18$ months, ${ }^{17}$ it is comparable to that of graphene on $\mathrm{Fe}$ surfaces with the added advantage that the insulating h-BN avoids the formation of a galvanic couple, ${ }^{54-56}$ which may otherwise accelerate corrosion of the underlying metal, as observed for graphene on $\mathrm{Cu}^{57}$ Furthermore, whereas 
bare $\mathrm{Fe}$ oxidizes almost immediately upon air exposure, we show that $\mathrm{h}-\mathrm{BN} / \mathrm{Fe}$ stacks offer a promising route for maintaining a reduced $\mathrm{Fe}$ surface when fabricating devices, where short air exposures between process steps including lithography, development, and lift off, as well as transfer of additional h-BN layers in air, can simplify fabrication or where oxidizing processing steps are required (e.g., atomic-layer/ pulsed-laser deposition of oxides, ${ }^{22,58}$ oxygen plasma to remove organic contaminants). Indeed, such integrated processing has been demonstrated by the incorporation of h-BN grown by $\mathrm{CVD}$ on $\mathrm{Fe}$ electrodes into functional magnetic tunnel junctions, ${ }^{6}$ where $\mathrm{h}-\mathrm{BN}$ serves as both a tunnel barrier and an oxidation barrier for Fe. Furthermore, the thermochemical stability of h-BN is expected to be particularly advantageous in aggressive processing environments, where other $2 \mathrm{D}$ materials would be damaged or removed. ${ }^{49-51}$

\section{CONCLUSIONS}

In summary, we have shown that single-layer h-BN grown on polycrystalline $\mathrm{Fe}$ foils protects the covered $\mathrm{Fe}$ surface from oxidation during exposure to moist air for more than $40 \mathrm{~h}$ at room temperature, whereas uncovered $\mathrm{Fe}$ regions become oxidized. This protection is attributed to the strong interaction between h-BN and the Fe substrate, which suppresses the rapid intercalation of oxidizing species along the interface between them. Over the longer term, slow bulk oxidation of Fe proceeds through the oxide layers formed in the vicinity of h-BN edges and defects, with the $\mathrm{Fe}$ eventually becoming oxidized throughout. The behavior observed is in excellent agreement with the model developed by Weatherup et al. to explain the passivation behavior of different graphene-covered metals, ${ }^{17}$ demonstrating that this general rationale can be extended to other $2 \mathrm{D}$ materials. We confirm that $\mathrm{h}-\mathrm{BN}$ remains stable during air exposure, with no oxidation of the h-BN detected. On postannealing in vacuum, Fe can be fully reduced, whereas h-BN remains largely intact, confirming that even after extended air exposures the reduced $\mathrm{h}-\mathrm{BN} / \mathrm{Fe}$ stack can be recovered. This annealing is found to induce some defects in h$\mathrm{BN}$ due to catalytic etching by the Fe support, indicating that the thermal stability of h-BN can be affected by the substrate on which it sits. Our results highlight the potential of h-BN films for preserving the surface of ferromagnetic electrodes in a reduced state during processing under oxidizing environments, which is particularly relevant to the transfer-free integration of $\mathrm{h}-\mathrm{BN}$ in devices such as MTJs.

\section{METHODS}

h-BN Growth. CVD growth is performed using a recipe previously reported. ${ }^{11}$ As-received Fe foil $(0.1 \mathrm{~mm}$, Alfa Aesar, $99.99 \%$ purity $)$ is used as the catalyst substrate for the growth of h-BN domains and films in a customized Aixtron BM3 cold-wall reactor (base pressure $<10^{-6}$ mbar $)$, using $\mathrm{B}_{3} \mathrm{~N}_{3} \mathrm{H}_{6}\left(6 \times 10^{-4} \mathrm{mbar}\right.$, FluoroChem, $>97 \%$ purity) as the precursor at $\sim 900{ }^{\circ} \mathrm{C}$. The samples are typically heated in $4 \mathrm{mbar}$ of $\mathrm{NH}_{3}$ at $100{ }^{\circ} \mathrm{C} / \mathrm{min}$ up to $750{ }^{\circ} \mathrm{C}$ and then at $50{ }^{\circ} \mathrm{C} / \mathrm{min}$ up to $\sim 900{ }^{\circ} \mathrm{C}$. Immediately after reaching $\sim 900{ }^{\circ} \mathrm{C}, \mathrm{NH}_{3}$ is removed. $\mathrm{B}_{3} \mathrm{~N}_{3} \mathrm{H}_{6}$ is dosed into the chamber through a leak valve (from a liquid reservoir), and after growth, the $\mathrm{B}_{3} \mathrm{~N}_{3} \mathrm{H}_{6}$ leak valve is closed and the heater is turned off. Samples are cooled in vacuum by immediately switching off the heater (cooling rate $\sim 300{ }^{\circ} \mathrm{C} / \mathrm{min}$ down to $\sim 500^{\circ} \mathrm{C}$, after which it slows down further).

Transfer. For optical microscopy and Raman spectroscopy, we transfer the h-BN using an electrochemical bubbling method. ${ }^{59} \mathrm{We}$ perform the transfer by spin-coating a support layer of poly(methyl methacrylate) (PMMA) onto the sample surface. The sample is placed in a $\mathrm{NaOH}$ bath $(1 \mathrm{M})$, and a negative voltage is applied relative to a Pt electrode, which causes $\mathrm{H}_{2}$ bubbles to evolve at the h-BN/Fe interface, detaching the film from the substrate. The PMMA/h-BN film is rinsed in deionized water and scooped onto a $\mathrm{SiO}_{2}(300 \mathrm{~nm}) / \mathrm{Si}$ wafer where it is left to dry. PMMA is removed by immersing the sample in acetone for $\sim 12 \mathrm{~h}$, followed by a rinse in IPA. The samples are annealed in vacuum at $500{ }^{\circ} \mathrm{C}$ for $1 \mathrm{~h}$ to remove residual PMMA. ${ }^{16}$

Characterization. For the ex situ characterization of h-BN on the catalyst, we use scanning electron microscopy (SEM, Zeiss SigmaVP, 2 $\mathrm{kV})$. Optical images are acquired using a Nikon eclipse ME600L microscope and Raman measurements are performed with a Renishaw In-Via microscope using a $100 \times$ objective and $532 \mathrm{~nm}$ laser excitation.

$\mathrm{XP}$ spectra of the $\mathrm{Fe} 2 \mathrm{p}_{3 / 2}$ core level were collected using a Thermo Scientific ESCALAB 250Xi at an operating pressure $<10^{-10} \mathrm{mbar}$. Xray illumination was provided by a microfocused, monochromated $\mathrm{Al}$ $\mathrm{K} \alpha$ source with a photon energy of $1486.6 \mathrm{eV}$ and a spot size of $\sim 150$ $\mu \mathrm{m}$ diameter. The emitted photoelectrons were collected by a hemispherical energy analyzer with a spectral resolution of $\pm 0.1 \mathrm{eV}$.

The X-ray photoelectron microscopy/microspectroscopy measurements were carried out with the SPEM instrument at the Escamicroscopy beamline of the Elettra synchrotron facility (Trieste, Italy). The X-ray beam was focused to $\sim 130 \mathrm{~nm}$ by a Fresnel zone plate and an order sorting aperture. For both imaging and submicron spectroscopy, a SPECS-PHOIBOS 100 hemispherical electron energy analyzer with an in-house customized multichannel plate detector was used. A photon energy of $1102 \mathrm{eV}$ was employed. The SPEM imaging mode can map the photoelectrons emitted within a selected kinetic energy window by scanning the specimen with respect to the X-ray beam. The SPEM microspectroscopy mode is the typical energy scanning mode employed in any standard XPS system, using a $130 \mathrm{~nm}$ $\mathrm{X}$-ray spot size. All binding energies are referenced to measured Fermi edges.

\section{ASSOCIATED CONTENT}

\section{Supporting Information}

The Supporting Information is available free of charge on the ACS Publications website at DOI: 10.1021/acsami.7b08717.

SEM image of Fe oxidation along specific channels in the h-BN film; XP spectra of the B 1s core level; SPEM chemical maps acquired with the B 1s, N 1s core-level energies; and XPS data showing the reduction of an $\mathrm{Fe}$ foil during annealing in vacuum (PDF)

\section{AUTHOR INFORMATION}

\section{Corresponding Author}

*E-mail: rsw31@cam.ac.uk.

ORCID $\odot$

Adrianus I. Aria: 0000-0002-6305-3906

Hikmet Sezen: 0000-0002-5438-3305

Stephan Hofmann: 0000-0001-6375-1459

Robert S. Weatherup: 0000-0002-3993-9045

Author Contributions

"S.C. and M.-B.M. have contributed equally.

Notes

The authors declare no competing financial interest.

\section{ACKNOWLEDGMENTS}

S.C. and L.D. acknowledge EPSRC Doctoral Training Awards. H.S. acknowledges a research fellowship from the Japanese Society for the Promotion of Science (JSPS). S.H. acknowledges funding from ERC grant InsituNANO (no. 279342). This research was partially supported by the EUFP7 Work Programme under grant GRAFOL (project reference 285275) and EPSRC under grant GRAPHTED (project reference EP/ 
K016636/1). R.S.W. acknowledges a Research Fellowship from St. John's College, Cambridge, and a Marie Skłodowska-Curie Individual Fellowship (Global) under grant ARTIST (no. 656870) from the European Union's Horizon 2020 research and innovation programme. We acknowledge the Elettra Sincrotrone Trieste storage ring for provision of synchrotron radiation at the Escamicroscopy beamline and thank the Elettra staff for continuous support of our experiments.

\section{REFERENCES}

(1) Dean, C. R.; Young, A. F.; Meric, I.; Lee, C.; Wang, L.; Sorgenfrei, S.; Watanabe, K.; Taniguchi, T.; Kim, P.; Shepard, K. L.; Hone, J. Boron Nitride Substrates for High-Quality Graphene Electronics. Nat. Nanotechnol. 2010, 5, 722-726.

(2) Bunch, J. S.; Verbridge, S. S.; Alden, J. S.; van der Zande, A. M.; Parpia, J. M.; Craighead, H. G.; McEuen, P. L. Impermeable Atomic Membranes from Graphene Sheets. Nano Lett. 2008, 8, 2458-2462.

(3) Liu, Z.; Gong, Y.; Zhou, W.; Ma, L.; Yu, J.; Idrobo, J. C.; Jung, J.; MacDonald, A. H.; Vajtai, R.; Lou, J.; Ajayan, P. M. Ultrathin HighTemperature Oxidation-Resistant Coatings of Hexagonal Boron Nitride. Nat. Commun. 2013, 4, No. 2541.

(4) Li, L. H.; Cervenka, J.; Watanabe, K.; Taniguchi, T.; Chen, Y. Strong Oxidation Resistance of Atomically Thin Boron Nitride Nanosheets. ACS Nano 2014, 8, 1457-1462.

(5) Ferrari, A. C.; Bonaccorso, F.; Falko, V.; Novoselov, K. S.; Roche, S.; Bøggild, P.; Borini, S.; Koppens, F.; Palermo, V.; Pugno, N.; Garrido, J. A.; Sordan, R.; Bianco, A.; Ballerini, L.; Prato, M.; Lidorikis, E.; Kivioja, J.; Marinelli, C.; Ryhänen, T.; Morpurgo, A.; Coleman, J. N.; Nicolosi, V.; Colombo, L.; Fert, A.; Garcia-Hernandez, M.; Bachtold, A.; Schneider, G. F.; Guinea, F.; Dekker, C.; Barbone, M.; Galiotis, C.; Grigorenko, A.; Konstantatos, G.; Kis, A.; Katsnelson, M.; Beenakker, C. W. J.; Vandersypen, L.; Loiseau, A.; Morandi, V.; Neumaier, D.; Treossi, E.; Pellegrini, V.; Polini, M.; Tredicucci, A.; Williams, G. M.; Hong, B. H.; Ahn, J. H.; Kim, J. M.; Zirath, H.; van Wees, B. J.; van der Zant, H.; Occhipinti, L.; Di Matteo, A.; Kinloch, I. A.; Seyller, T.; Quesnel, E.; Feng, X.; Teo, K.; Rupesinghe, N.; Hakonen, P.; Neil, S. R. T.; Tannock, Q.; Löfwander, T.; Kinaret, J. Science and Technology Roadmap for Graphene, Related TwoDimensional Crystals, and Hybrid Systems. Nanoscale 2015, 7, 45984810.

(6) Piquemal-Banci, M.; Galceran, R.; Caneva, S.; Martin, M.-B.; Weatherup, R. S.; Kidambi, P. R.; Bouzehouane, K.; Xavier, S.; Anane, A.; Petroff, F.; Fert, A.; Robertson, J.; Hofmann, S.; Dlubak, B.; Seneor, P. Magnetic Tunnel Junctions with Monolayer Hexagonal Boron Nitride Tunnel Barriers. Appl. Phys. Lett. 2016, 108, No. 102404.

(7) Hao, Y.; Bharathi, M.; Wang, L.; Liu, Y.; Chen, H.; Nie, S.; Wang, X.; Chou, H.; Tan, C.; Fallahazad, B.; Ramanarayan, H.; Magnuson, C. W.; Tutuc, E.; Yakobson, B. I.; McCarty, K. F.; Zhang, Y.-W.; Kim, P.; Hone, J.; Colombo, L.; Ruoff, R. S. The Role of Surface Oxygen in the Growth of Large Single-Crystal Graphene on Copper. Science 2013, $342,720-723$.

(8) Weatherup, R. S.; Shahani, A. J.; Wang, Z.-J.; Mingard, K.; Pollard, A. J.; Willinger, M. G.; Schloegl, R.; Voorhees, P. W.; Hofmann, S. In Situ Graphene Growth Dynamics on Polycrystalline Catalyst Foils. Nano Lett. 2016, 16, 6196-6206.

(9) Nagashima, A.; Tejima, N.; Oshima, C. Electronic States of the Pristine and Alkali-Metal-Intercalated Monolayer graphite/Ni(111) Systems. Phys. Rev. B 1994, 50, 17487-17495.

(10) Caneva, S.; Weatherup, R. S.; Bayer, B.; Brennan, B.; Spencer, S. J.; Mingard, K.; Cabrero-Vilatela, A.; Baehtz, C.; Pollard, A. J.; Hofmann, S. Nucleation Control for Large, Single Crystalline Domains of Monolayer Hexagonal Boron Nitride via Si-Doped Fe Catalysts. Nano Lett. 2015, 15, 1867-1875.

(11) Caneva, S.; Weatherup, R. S.; Bayer, B. C.; Blume, R.; CabreroVilatela, A.; Braeuninger-Weimer, P.; Martin, M.-B.; Wang, R.; Baehtz, C.; Schlögl, R.; Meyer, J. C.; Hofmann, S. Controlling Catalyst Bulk
Reservoir Effects for Monolayer Hexagonal Boron Nitride CVD. Nano Lett. 2016, 16, 1250-1261.

(12) Lu, G.; Wu, T.; Yuan, Q.; Wang, H.; Wang, H.; Ding, F.; Xie, X.; Jiang, M. Synthesis of Large Single-Crystal Hexagonal Boron Nitride Grains on Cu-Ni Alloy. Nat. Commun. 2015, 6, No. 6160.

(13) Stehle, Y.; Meyer, H. M.; Unocic, R. R.; Kidder, M.; Polizos, G.; Datskos, P. G.; Jackson, R.; Smirnov, S. N.; Vlassiouk, I. V. Synthesis of Hexagonal Boron Nitride Monolayer: Control of Nucleation and Crystal Morphology. Chem. Mater. 2015, 27, 8041-8047.

(14) Oshima, C.; Nagashima, A. Ultra-Thin Epitaxial Films of Graphite and Hexagonal Boron Nitride on Solid Surfaces. J. Phys.: Condens. Matter 1997, 9, 1-20.

(15) Wang, R.; Whelan, P. R.; Braeuninger-Weimer, P.; Tappertzhofen, S.; Alexander-Webber, J. A.; Van-Veldhoven, Z. A.; Kidambi, P. R.; Jessen, B. S.; Booth, T. J.; Boggild, P.; Hofmann, S. Catalyst Interface Engineering for Improved 2D Film Lift-Off and Transfer. ACS Appl. Mater. Interfaces 2016, 8, 33072.

(16) Kratzer, M.; Bayer, B. C.; Kidambi, P. R.; Matković, A.; Gajić, R.; Cabrero, A.; Weatherup, R. S.; Hofmann, S.; Teichert, C. Effects of Polymethylmethacrylate-Transfer Residues on the Growth of Organic Semiconductor Molecules on Chemical Vapor Deposited Graphene. Appl. Phys. Lett. 2015, 106, No. 103101.

(17) Weatherup, R. S.; D’Arsié, L.; Cabrero-Vilatela, A.; Caneva, S.; Blume, R; Robertson, J.; Schlögl, R.; Hofmann, S. Long-Term Passivation of Strongly Interacting Metals with Single-Layer Graphene. J. Am. Chem. Soc. 2015, 137, 14358-14366.

(18) Dedkov, Y. S.; Fonin, M.; Rüdiger, U.; Laubschat, C. GrapheneProtected Iron Layer on $\mathrm{Ni}(111)$. Appl. Phys. Lett. 2008, 93, No. 022509.

(19) Dahal, A.; Batzill, M. Graphene-Nickel Interfaces: A Review. Nanoscale 2014, 6, 2548-2562.

(20) Weatherup, R. S.; Amara, H.; Blume, R.; Dlubak, B.; Bayer, B. C.; Diarra, M.; Bahri, M.; Cabrero-vilatela, A.; Caneva, S.; Kidambi, P. R.; Martin, M.; Deranlot, C.; Seneor, P.; Schloegl, R.; Bichara, C.; Hofmann, S.; Ducastelle, F. Interdependency of Subsurface Carbon Distribution and Graphene-Catalyst Interactions. J. Am. Chem. Soc. 2014, 136, 13698-13708.

(21) Dlubak, B.; Martin, M.-B.; Weatherup, R. S.; Yang, H.; Deranlot, C.; Blume, R.; Schloegl, R.; Fert, A.; Anane, A.; Hofmann, S.; Seneor, P.; Robertson, J. Graphene-Passivated Nickel as an OxidationResistant Electrode for Spintronics. ACS Nano 2012, 6, 10930-10934.

(22) Martin, M.-B.; Dlubak, B.; Weatherup, R. S.; Yang, H.; Deranlot, C.; Bouzehouane, K.; Petroff, F.; Anane, A.; Hofmann, S.; Robertson, J.; Fert, A.; Seneor, P. Sub-Nanometer Atomic Layer Deposition for Spintronics in Magnetic Tunnel Junctions Based on Graphene SpinFiltering Membranes. ACS Nano 2014, 8, 7890-7895.

(23) Martin, M.-B.; Dlubak, B.; Weatherup, R. S.; Piquemal-Banci, M.; Yang, H.; Blume, R.; Schloegl, R.; Collin, S.; Petroff, F.; Hofmann, S.; Robertson, J.; Anane, A.; Fert, A.; Seneor, P. Protecting Nickel with Graphene Spin-Filtering Membranes: A Single Layer Is Enough. Appl. Phys. Lett. 2015, 107, No. 012408.

(24) Kumar, S.; McEvoy, N.; Kim, H.-Y.; Lee, K.; Peltekis, N.; Rezvani, E.; Nolan, H.; Weidlich, A.; Daly, R.; Duesberg, G. S. CVD Growth and Processing of Graphene for Electronic Applications. Phys. Status Solidi B 2011, 248, 2604-2608.

(25) Britnell, L.; Gorbachev, R. V.; Jalil, R; Belle, B. D.; Schedin, F.; Katsnelson, M. I.; Eaves, L.; Morozov, S. V.; Mayorov, A. S.; Peres, N. M. R.; Neto, A. H. C.; Leist, J.; Geim, A. K.; Ponomarenko, L. A.; Novoselov, K. S. Electron Tunneling through Ultrathin Boron Nitride Crystalline Barriers. Nano Lett. 2012, 12, 1707-1710.

(26) Kirczenow, G. Ideal Spin Filters: A Theoretical Study of Electron Transmission through Ordered and Disordered Interfaces between Ferromagnetic Metals and Semiconductors. Phys. Rev. B 2001, 63, No. 054422.

(27) Dankert, A.; Venkata Kamalakar, M.; Wajid, A.; Patel, R. S.; Dash, S. P. Tunnel Magnetoresistance with Atomically Thin TwoDimensional Hexagonal Boron Nitride Barriers. Nano Res. 2015, 8, $1357-1364$ 
(28) Kamalakar, M. V.; Dankert, A.; Kelly, P. J.; Dash, S. P. Inversion of Spin Signal and Spin Filtering in Ferromagnet|Hexagonal Boron Nitride-Graphene van Der Waals Heterostructures. Sci. Rep. 2016, 6, No. 21168.

(29) Asshoff, P. U.; Sambricio, J. L.; Rooney, A. P.; Slizovskiy, S.; Mishchenko, A.; Rakowski, A. M.; Hill, E. W.; Geim, A. K.; Haigh, S. J.; Fal'ko, V. I.; Vera-Marun, I. J.; Grigorieva, I. V. Magnetoresistance of Vertical Co-Graphene-NiFe Junctions Controlled by Charge Transfer and Proximity-Induced Spin Splitting in Graphene. 2D Mater. 2017, 4, No. 031004.

(30) Gibb, A. L.; Alem, N.; Chen, J.; Erickson, K. J.; Ciston, J.; Gautam, A.; Linck, M.; Zettl, A. Atomic Resolution Imaging of Grain Boundary Defects in Monolayer. J. Am. Chem. Soc. 2013, 135, 67586761.

(31) Weatherup, R. S.; Dlubak, B.; Hofmann, S. Kinetic Control of Catalytic CVD for High Quality Graphene at Low Temperatures. ACS Nano 2012, 6, 9996-10003.

(32) Cabrero-Vilatela, A.; Weatherup, R. S.; Braeuninger-Weimer, P.; Caneva, S.; Hofmann, S. Towards a General Growth Model for Graphene CVD on Transition Metal Catalysts. Nanoscale 2016, 8, 2149-2158.

(33) Müller, F.; Hüfner, S.; Sachdev, H.; Gsell, S.; Schreck, M. Epitaxial Growth of Hexagonal Boron Nitride Monolayers by a ThreeStep Boration-Oxidation-Nitration Process. Phys. Rev. B: Condens. Matter Mater. Phys. 2010, 82, No. 075405.

(34) Welsh, I. D.; Sherwood, P. M. A. Photoemission and ElectronicStructure of FeOOH - Distinguishing Between Oxide and Oxyhydroxide. Phys. Rev. B: Condens. Matter Mater. Phys. 1989, 40, 63866392.

(35) Blume, R.; Kidambi, P. R.; Bayer, B. C.; Weatherup, R. S.; Wang, Z.-J.; Weinberg, G.; Willinger, M.-G.; Greiner, M.; Hofmann, S.; Knop-Gericke, A.; Schlögl, R. The Influence of Intercalated Oxygen on the Properties of Graphene on Polycrystalline $\mathrm{Cu}$ under Various Environmental Conditions. Phys. Chem. Chem. Phys. 2014, 16, 2598926003.

(36) Usachov, D.; Adamchuk, V. K.; Haberer, D.; Grüneis, A.; Sachdev, H.; Preobrajenski, A. B.; Laubschat, C.; Vyalikh, D. V. Quasifreestanding Single-Layer Hexagonal Boron Nitride as a Substrate for Graphene Synthesis. Phys. Rev. B 2010, 82, No. 075415.

(37) Preobrajenski, A. B.; Vinogradov, A. S.; Mårtensson, N. Monolayer of $\mathrm{H}-\mathrm{BN}$ Chemisorbed on $\mathrm{Cu}(111)$ and $\mathrm{Ni}(111)$ : The Role of the Transition Metal 3d States. Surf. Sci. 2005, 582, 21-30.

(38) Nagashima, A.; Tejima, N.; Gamou, Y.; Kawai, T.; Oshima, C. Electronic Structure of Monolayer Hexagonal Boron Nitride Physisorbed on Metal Surfaces. Phys. Rev. Lett. 1995, 75, 3918-3921.

(39) Liu, N.; Pan, Z.; Fu, L.; Zhang, C.; Dai, B.; Liu, Z. The Origin of Wrinkles on Transferred Graphene. Nano Res. 2011, 4, 996-1004.

(40) Ashby, M. F.; Jones, D. R. H. Oxidation and Corrosion. In Engineering Materials I: An Introduction to Properties, Applications, and Design; Elsevier: Oxford, 2012; pp 385-400.

(41) Jagadeesh, R. V.; Surkus, A.-E.; Junge, H.; Pohl, M.-M.; Radnik, J.; Rabeah, J.; Huan, H.; Schunemann, V.; Bruckner, A.; Beller, M. Nanoscale Fe2O3-Based Catalysts for Selective Hydrogenation of Nitroarenes to Anilines. Science 2013, 342, 1073-1076.

(42) Luo, W.; Zafeiratos, S. A Brief Review of the Synthesis and Catalytic Applications of Graphene-Coated Oxides. ChemCatChem 2017, 9, 2432-2442.

(43) Kravets, V. G.; Jalil, R.; Kim, Y.-J.; Ansell, D.; Aznakayeva, D. E.; Thackray, B.; Britnell, L.; Belle, B. D.; Withers, F.; Radko, I. P.; Han, Z.; Bozhevolnyi, S. I.; Novoselov, K. S.; Geim, A. K.; Grigorenko, A. N. Graphene-Protected Copper and Silver Plasmonics. Sci. Rep. 2014, 4, No. 5517.

(44) Grosvenor, A. P.; Kobe, B. A.; McIntyre, N. S. Examination of the Oxidation of Iron by Oxygen Using X-Ray Photoelectron Spectroscopy and QUASES. Surf. Sci. 2004, 565, 151-162.

(45) Grosvenor, A. P.; Kobe, B. A.; McIntyre, N. S. Studies of the Oxidation of Iron by Water Vapour Using X-Ray Photoelectron Spectroscopy and QUASES. Surf. Sci. 2004, 572, 217-227.
(46) Zhang, Y.; Weng, X.; Li, H.; Li, H.; Wei, M.; Xiao, J.; Liu, Z.; Chen, M.; Fu, Q.; Bao, X. Hexagonal Boron Nitride Cover on Pt(111): A New Route to Tune Molecule-Metal Interaction and Metal-Catalyzed Reactions. Nano Lett. 2015, 15, 3616-3623.

(47) Brugger, T.; Ma, H.; Iannuzzi, M.; Berner, S.; Winkler, A.; Hutter, J.; Osterwalder, J.; Greber, T. Nanotexture Switching of SingleLayer Hexagonal Boron Nitride on Rhodium by Intercalation of Hydrogen Atoms. Angew. Chem. - Int. Ed. 2010, 49, 6120-6124.

(48) Sutter, P.; Sadowski, J. T.; Sutter, E. A. Chemistry under Cover: Tuning Metal-Graphene Interaction by Reactive Intercalation. J. Am. Chem. Soc. 2010, 132, 8175-8179.

(49) Gao, J.; Li, B.; Tan, J.; Chow, P.; Lu, T. M.; Koratkar, N. Aging of Transition Metal Dichalcogenide Monolayers. ACS Nano 2016, 10, 2628-2635.

(50) Favron, A.; Gaufrès, E.; Fossard, F.; Phaneuf-L'Heureux, A.-L.; Tang, N. Y.-W.; Lévesque, P. L.; Loiseau, A.; Leonelli, R.; Francoeur, S.; Martel, R. Photooxidation and Quantum Confinement Effects in Exfoliated Black Phosphorus. Nat. Mater. 2015, 14, 826-832.

(51) Rong, Y.; He, K.; Pacios, M.; Robertson, A. W.; Bhaskaran, H.; Warner, J. H. Controlled Preferential Oxidation of Grain Boundaries in Monolayer Tungsten Disulfide for Direct Optical Imaging. ACS Nano 2015, 9, 3695-3703.

(52) Kidambi, P. R.; Blume, R.; Kling, J.; Wagner, J. B.; Baehtz, C.; Weatherup, R. S.; Schloegl, R.; Bayer, B. C.; Hofmann, S. In Situ Observations during Chemical Vapor Deposition of Hexagonal Boron Nitride on Polycrystalline Copper. Chem. Mater. 2014, 26, 63806392.

(53) Simonov, K. A.; Vinogradov, N. A.; Ng, M. L.; Vinogradov, A. S.; Mårtensson, N.; Preobrajenski, A. B. Controllable Oxidation of $\mathrm{H}-$ BN Monolayer on Ir(111) Studied by Core-Level Spectroscopies. Surf. Sci. 2012, 606, 564-570.

(54) Galbiati, M.; Stoot, A. C.; Mackenzie, D. M. A.; Bøggild, P.; Camilli, L. Real-Time Oxide Evolution of Copper Protected by Graphene and Boron Nitride Barriers. Sci. Rep. 2017, 7, No. 39770.

(55) Shen, L.; Zhao, Y.; Wanf, Y.; Song, R.; Yao, Q.; Chen, S.; Chai, Y. Long-Term Corrosion Barrier with Insulating Boron Nitride Monolayer. J. Mater. Chem. A 2016, 4, 5044-5050.

(56) Li, X.; Yin, J.; Zhou, J.; Guo, W. Large Area Hexagonal Boron Nitride Monolayer as Efficient Atomically Thick Insulating Coating against Friction and Oxidation. Nanotechnology 2014, 25, No. 105701.

(57) Schriver, M.; Regan, W.; Gannett, W. J.; Zaniewski, A. M.; Crommie, M. F.; Zettl, A. Graphene as a Long-Term Metal Oxidation Barrier: Worse than Nothing. ACS Nano 2013, 7, 5763-5768.

(58) Dlubak, B.; Kidambi, P. R.; Weatherup, R. S.; Hofmann, S.; Robertson, J. Substrate-Assisted Nucleation of Ultra-Thin Dielectric Layers on Graphene by Atomic Layer Deposition. Appl. Phys. Lett. 2012, 100, No. 173113.

(59) Gao, L.; Ren, W.; Xu, H.; Jin, L.; Wang, Z.; Ma, T.; Ma, L.-P.; Zhang, Z.; Fu, Q.; Peng, L.-M.; Bao, X.; Cheng, H.-M. Repeated Growth and Bubbling Transfer of Graphene with Millimetre-Size Single-Crystal Grains Using Platinum. Nat. Commun. 2012, 3, No. 699.

(60) Spizzirri, P. G.; Rubanov, S.; Gauja, E.; Prawer, S. Nano-Raman Spectroscopy of Silicon Surfaces. Mater. Forum 2008, 32, 161-166. 